\title{
O papel dos produtores públicos de medicamentos e ações estratégicas na pandemia da Covid-19
}

\author{
The role of public producers of medicines and strategic actions in the \\ Covid-19 pandemic
}

Daniela Rangel Affonso Fernandes', Carlos Augusto Grabois Gadelha², Jose Manuel Santos de Varge Maldonado ${ }^{2}$

DOI: $10.1590 / 0103-1104202213201$

RESUMO Na pandemia da Covid-19, os laboratórios farmacêuticos públicos adquiriram maior visibilidade em consequência de suas iniciativas para enfrentamento da doença e manutenção dos diversos programas da assistência farmacêutica. $\mathrm{O}$ artigo objetivou analisar as atividades cotidianas desses laboratórios durante a pandemia, a fim de compreender seu caráter estratégico para o Sistema Único de Saúde (SUS), destacando os Institutos Butantan, Bio-Manguinhos e Farmanguinhos, haja vista o protagonismo em relação a vacinas e medicamentos. Mediante estudo de caso múltiplo, com abordagem qualitativa-descritiva, apresentou dados que indicam a mudança de perfil que vêm demonstrando nas últimas décadas. Identificaram-se as ações de maior relevância no enfrentamento da pandemia, tendo, como data de corte, julho de 2021. Como resultados, trouxe informações atuais sobre suas atividades e produtos, estágio das Parcerias para Desenvolvimento Produtivo, quadro funcional, fragilidades (internas e externas) e desafios atuais. Conclui-se pela importância dessas instituições do Estado para a garantia do acesso universal do SUS e desenvolvimento e produção de medicamentos e produtos de saúde essenciais, envolvendo desde os mais básicos até os de maior complexidade e valor agregado. Destarte, identifica-se a necessidade de uma agenda de mudanças, visando sustentabilidade em longo prazo e ampliação da contribuição para fortalecimento do Complexo Econômico-Industrial da Saúde.

PALAVRAS-CHAVE Produção pública. Vacinas. Medicamentos. Covid-19. Complexo Econômico-Industrial da Saúde.

ABSTRACT In the Covid-19 pandemic, public pharmaceutical laboratories gained greater visibility because of their initiatives to fight the disease and maintain the various pharmaceutical assistance programs. The article aimed to analyze their daily activities during the pandemic, to understand their strategic character for the Unified Health System (SUS), highlighting the Butantan, Bio-Manguinhos, and Farmanguinhos Institutes, given their leading role in relation to vaccines and medicines, respectively. Through a multiple case study, with a qualitative-descriptive approach, it presented data that indicate the change in profile that has been demonstrated in recent decades. The most relevant actions in combating the pandemic were identified, with

Fundação Oswaldo Cruz (Fiocruz), Instituto de Tecnologia em Fármacos (ITF) - Rio de Janeiro (RJ), Brasil. daniela.fernandes@far. fiocruz.br

2 Fundação Oswaldo Cruz (Fiocruz), Escola Naciona de Saúde Pública Sergio Arouca (Ensp) - Rio de Janeiro (RJ), Brasil. the cut-off date of July 2021. As a result, it brought current information about its activities and products, stage of the Partnerships for Productive Development, staff, weaknesses (internal and external), and current challenges. It is concluded that these State Institutions are important to guarantee universal access to the SUS and the development and production of essential medicines and health products, ranging from the most basic to those with greater complexity and added value. Thus, the need for an agenda of changes is identified, aiming at long-term sustainability and expanding the contribution to strengthen the Economic-Industrial Health Complex.

KEYWORDS Public production. Vaccines. Medicines. Covid-19. Health Economic-Industrial Complex. 


\section{Introdução}

O acesso a medicamentos e produtos de saúde é um importante indicador da qualidade de vida de uma população․․ Apesar de sua relevância para a saúde global, a pesquisa, o desenvolvimento e a produção concentram-se em poucas empresas líderes, geralmente de países desenvolvidos ${ }^{2}$, voltados a interesses comerciais. Esse fato pode acarretar exclusão de acesso à saúde para significativas parcelas da humanidade, designadas populações negligenciadas.

No Brasil, desde que o direito universal à saúde foi conquistado, na Constituição Federal em 1988, ampliou-se o acesso a serviços e medicamentos, favorecendo melhorias nas condições de saúde e na economia do País ${ }^{3}$ e promovendo mudanças no perfil epidemiológico da população ${ }^{4}$. Grandes transformações ocorreram desde então, a exemplo de mudanças na concepção dos princípios do Sistema Único de Saúde (SUS), na organização e na relação do governo federal com os estados, municípios e sociedade civil, assim como nos programas da assistência farmacêutica ${ }^{5}$.

A sociedade em mudança influenciou a implantação de políticas públicas, visando, entre outros pontos, à garantia de acesso a medicamentos e produtos de saúde. Nesse contexto, o Estado, ainda que de forma incipiente, tem fomentado o desenvolvimento da base produtiva e tecnológica da saúde e do sistema de inovação local. O apoio tem-se dado por meio de financiamentos, medidas protecionistas e implementações de políticas industriais e de saúde, podendo-se destacar: a Política Nacional de Medicamentos (1998), a Política Industrial, Tecnológica e de Comércio Exterior (PITCE) (2003), a Política de Desenvolvimento Produtivo (2008), o Plano Brasil Maior (2011) e a Estratégia Nacional para Ciência, Tecnologia e Inovação (ENCTI) (2012).

O Complexo Econômico-Industrial da Saúde (Ceis), cujo conceito foi desenvolvido no início dos anos 2000 para captar a relação indissociável entre saúde e desenvolvimento, possui a indústria farmacêutica com maior peso relativo entre seus demais segmentos (equipamentos, materiais médicos, serviços) ${ }^{2}$. Tem como característica a presença de uma ampla rede de laboratórios públicos, voltados para atendimento quase exclusivo das necessidades do SUS em relação a medicamentos, soros, vacinas, kits de diagnóstico e produtos para a saúde.

Conhecidos como Laboratórios Farmacêuticos Oficiais (LFO), seus produtos e serviços os qualificam como estratégicos provedores dos programas de assistência farmacêutica e como importantes atores do desenvolvimento da ciência e tecnologia nacionais. $\mathrm{O}$ estudo teve como objetivo analisar a trajetória, os desafios e as potencialidades desses laboratórios, assim como identificar suas ações para enfrentamento da pandemia da Covid-19, em especial, do Instituto Butantan, do Instituto de Tecnologia em Imunobiológicos (Bio-Manguinhos) e do Instituto de Tecnologia em Fármacos (Farmanguinhos), contribuindo para a reflexão acerca do papel dessas Instituições de Estado na garantia do acesso universal do SUS e no fortalecimento do Ceis.

\section{Material e métodos}

$\mathrm{O}$ artigo estrutura-se em duas seções, uma geral e outra mais específica. A primeira refere-se à rede de laboratórios públicos, apresentando informações sobre seu histórico, perfil, situação das Parcerias para o Desenvolvimento Produtivo (PDP), seus desafios e fragilidades. A segunda parte concerne às ações realizadas pelos Institutos Butantan, Bio-Manguinhos e Farmanguinhos com vistas à prevenção, à detecção e à proteção da saúde nacional em relação a vacina e medicamentos contra a Covid-19. Optou-se por um estudo de caso múltiplo sobre três laboratórios oficiais, 
realizado por meio de uma pesquisa qualitativa, aliando a revisão bibliográfica à documental, por intermédio de uma abordagem descritiva. A escolha dos três institutos de destaque deve-se à liderança que exercem em suas áreas de atuação, sendo respectivamente, de imunobiológicos, vacinas e medicamentos, e pela relevância de suas ações no enfrentamento da pandemia.

A pesquisa bibliográfica foi realizada nas bases de dados Biblioteca Virtual em Saúde (BVS) e na Scientific Electronic Library Online (SciELO), utilizando palavraschave como "laboratório oficial" e Ceis and desenvolvimento or acesso, buscando publicações de 2000 até hoje. Totalizaram 145 publicações encontradas, das quais foram selecionadas 21 , pelo conteúdo afeto aos institutos estudados. A partir do conteúdo das publicações selecionadas e com intuito de se aprofundar na investigação e obter informações pontuais e recentes, realizou-se uma pesquisa documental e de dados secundários, tendo, como data de corte, julho de 2021. A primeira baseou-se em relatórios e documentos oficiais dos institutos destacados; e a segunda, em portais eletrônicos do Ministério da Saúde (MS), Fundação Oswaldo Cruz (Fiocruz), Associação de Laboratórios Oficiais do Brasil (Alfob), Associação Brasileira das Indústrias de Química Fina, Biotecnologia e suas Especialidades (Abifina) e dos sítios eletrônicos dos 20 laboratórios oficiais em funcionamento atualmente. Para a construção do gráfico 1, foi feita a consulta ao banco de dados da Agência Nacional de Vigilância Sanitária (Anvisa) para confirmação do número de registros válidos para medicamentos; e para o quadro 2, relativo às PDP, utilizaram-se as planilhas mais recentes disponibilizadas pela Secretaria de Ciência, Tecnologia, Inovação e Insumos Estratégicos em Saúde (SCTIE) do MS, atualizadas até setembro de 2020.

\section{Resultados e discussão}

\section{Os Laboratórios Farmacêuticos Oficiais}

\section{UMA BREVE RETROSPECTIVA}

Os institutos tiveram sua origem voltada ao atendimento das demandas emergenciais e estruturais da assistência farmacêutica, visando à cobertura das lacunas existentes na produção nacional de vacinas e medicamentos essenciais ${ }^{6,7}$. Desde 1808, quando foi fundada a Botica Real Militar (atual Laboratório Químico Farmacêutico do Exército), até os dias atuais, têm provido a população brasileira de produtos indispensáveis à sua saúde, mantendo-se, mesmo diante de diversos cenários político-institucionais, como importantes atores para as políticas públicas de Estado ${ }^{6}$.

Ao longo do tempo, passaram a compor a política de assistência farmacêutica, deixando, gradativamente, de considerar apenas a demanda da atenção básica e passando a incluir medicamentos de distintas naturezas, como, por exemplo, os antirretrovirais. A relação desses medicamentos estendeu-se também para medicamentos de maior valor agregado e elevado conteúdo tecnológico, dando origem ao componente estratégico da assistência farmacêutica ${ }^{7}$.

O Instituto Butantan (1899), em São Paulo, e a Fiocruz (1900), no Rio de Janeiro, foram criados no contexto da epidemia de peste bubônica, visando à produção nacional de soro e vacina contra a doença, tendo em vista a dificuldade de aquisição do Instituto Pasteur, da França, onde o soro era produzido ${ }^{\mathbf{8}, 9}$. No início do século XX, foram criados o Laboratório Farmacêutico da Marinha (LFM), em 1906; a Fundação Ezequiel Dias (Funed), em 1907; e o Instituto Vital Brazil S.A. (IVB), em 1918. A expansão da rede de LFO no País coincidiu com 
um momento da adoção de políticas de orientação desenvolvimentista, pela necessidade de fortalecer a indústria químico-farmacêutica nacional. No período de 1964 a 1974, foram criados oito $\mathrm{LFO}^{10}$.

Até o início do século XX, suas atividades eram marcadas pela produção de medicamentos de uso tópico, vacinas e soros antipeçonhentos ${ }^{6}$. Na década de 1970, a criação da Central de Medicamentos (Ceme) levou esses laboratórios a atenderem grande parte da demanda de medicamentos na rede pública de saúde do País. Em 1976, foram criados: o laboratório Farmanguinhos, posteriormente elevado à condição de instituto, com a missão de desenvolver tecnologias de produção, adaptar tecnologias transferidas e elaborar produtos quimioterápicos e profiláticos para atender às necessidades dos programas de saúde e às exigências da Segurança Nacional ${ }^{\mathbf{8}}$; e Bio-Manguinhos, instituto voltado à promoção, ao desenvolvimento e à produção de imunobiológicos de interesse para a saúde pública ${ }^{9}$, ambos da Fiocruz.

Com a aprovação da Lei Orgânica da Saúde (Lei $n^{\circ}$ 8080/90), que instituiu o SUS, o paradigma incorporado da integralidade provocou a necessidade de ampliação da oferta local. Em 1998, com a Política Nacional de Medicamentos (PNM) - Portaria n ${ }^{\circ} 3916$-, os laboratórios foram induzidos a fornecer novos tipos de medicamentos 5 .

Em 2005, quando a política de descentralização da assistência farmacêutica foi mais fortemente observada (Portaria $\mathrm{n}^{\circ} 2.084 / 05$ ), deixando o MS de adquirir de forma centralizada muitos medicamentos utilizados na atenção básica, de atendimento das políticas de distribuição em massa, o que permitiu aos Munícipios e Estados comprarem diretamente de qualquer empresa farmacêutica, uma significativa redução de demanda ocorreu, ocasionando perda de mercado e baixo aproveitamento de suas capacidades instaladas e expertises tecnológicas ${ }^{11}$.

Nesse contexto, a missão dos produtores oficiais se ampliou. Suprindo falhas de mercado, passaram cada vez mais a absorver medicamentos e produtos de interesse de programas estratégicos de saúde pública, mesmo de demanda mais reduzida"1, como o Doenças Sexualmente Transmissíveis (DST)/Aids e o Programa Nacional de Imunização (PNI), os quais, por conta de práticas monopolistas e oligopolistas do mercado farmacêutico, possuem geralmente um alto custo, o que restringe o acesso ao SUS7,12.

\section{PERFIL E MISSÃO}

Atualmente, existem 20 LFO formando a Rede Brasileira de Produção Pública de Medicamentos (RBPPM), que foi criada em 2005 (Portaria GM/MS n².438) com objetivo de integrar e melhorar o desempenho do conjunto dos laboratórios públicos. Os LFO possuem como missão principal produzir medicamentos constantes na Relação Nacional de Medicamentos Essenciais (Rename) para atender à demanda do SUS. Alguns se diferenciam por sua capacidade instalada, como Farmanguinhos, Bio-Manguinhos e Butantan, tendo os dois últimos destaque em vacinas e imunobiológicos.

Suas vendas destinam-se quase exclusivamente ao MS e às Secretarias Estaduais e Municipais de Saúde, à exceção de BioManguinhos, ligado à Fiocruz, que participa de licitações internacionais da Organização Pan-Americana da Saúde (Opas) ${ }^{4}$. Possuem características, vínculos e portes diferentes, concentrando-se, em sua maioria, na região Sudeste (53\%). A maior parte está vinculada a governos estaduais, quatro são ligados a universidades, e cinco, a instituições federais.

Nem todos têm produção própria. A maioria atua em duas frentes: fornecimento de medicamentos produzidos internamente (sendo o detentor do registro ou local de fabricação); ou produzidos por terceiros, como no caso das PDP, durante o período de transferência tecnológica ${ }^{7}$. De acordo com dados extraídos do site da Anvisa ${ }^{\mathbf{1 3}}$, havia 290 medicamentos com registros válidos (gráfico 1), destacando-se 
nesse quantitativo a Fundação para o Remédio Popular (Furp), com 33\% do total, seguido por Farmanguinhos e Funed. Dos 20 laboratórios públicos, 14 (70\%) possuem medicamentos aprovados pela Anvisa. Desses medicamentos, $86 \%$ são de síntese química, e apenas $8 \%$ referem-se a produtos de origem biológica. O registro válido não significa sua necessária produção, mas indica que o LFO está apto a produzir caso haja demanda para o medicamento em questão ${ }^{\mathbf{1 4}}$.

Gráfico 1. Medicamentos com registros válidos por LFO

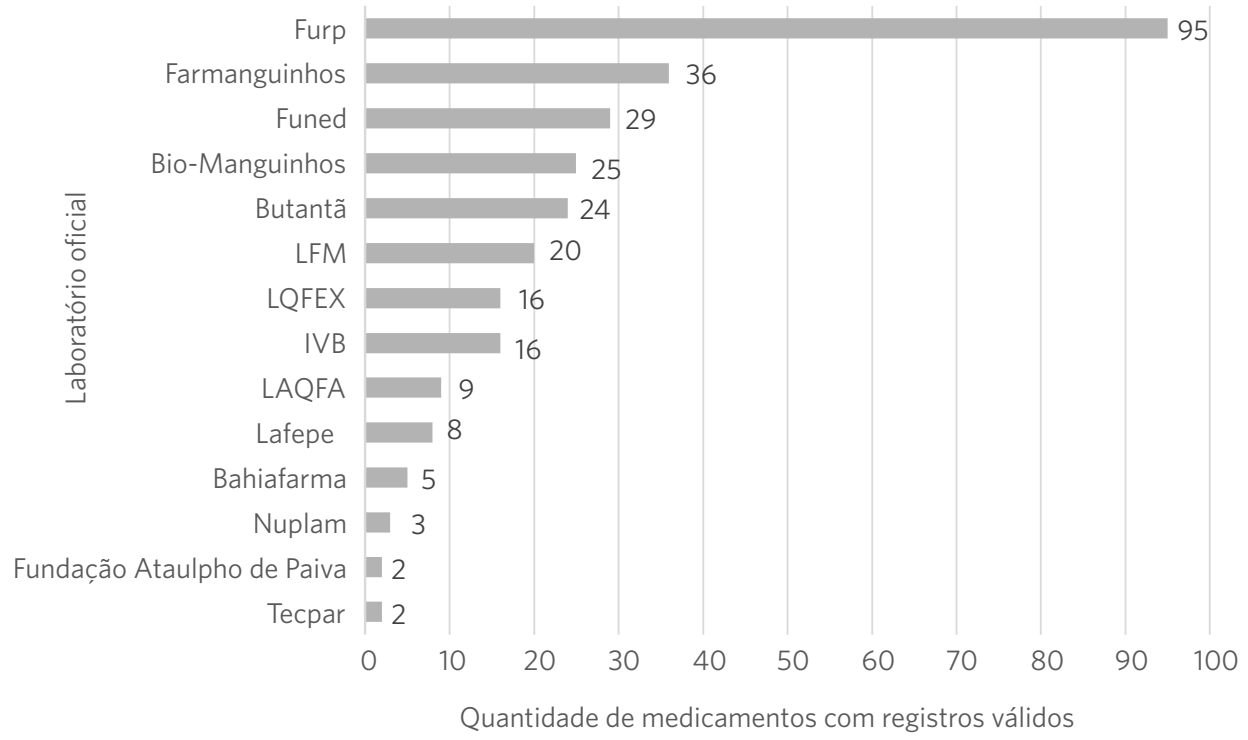

Fonte: elaboração própria a partir de informações extraídas do site da Anvisa ${ }^{\mathbf{6}} \mathrm{em}$ maio de 2021.

Cabe destacar que os LFO não atendem nem seria racional atenderem toda a Rename. A lista, criada em 1975, sofre revisões periódicas a cada dois anos; e para ter uma noção, a última lista disponibilizada pelo SUS, na edição de 2020, possui 916 itens $^{15}$. Diante disso, cabe ao governo, por meio de políticas públicas, priorizar os medicamentos a serem produzidos pelos produtores públicos ${ }^{16}$, evitando concorrência com as empresas privadas nacionais e multinacionais e criando condições para a produção de medicamentos de maior valor agregado, estratégicos para o SUS.

Além da atividade produtiva, os LFO têm o propósito de subsidiar a formulação de políticas públicas e participar da geração e difusão de conhecimentos em saúde, tendo como atividades a pesquisa e o desenvolvimento tecnológico.

\section{PRODUTOS}

Oferecem ao SUS um amplo portfólio de produtos voltados às necessidades da população, entre vacinas, soros, medicamentos sintéticos e biológicos e produtos para saúde (quadro 1), atendendo cerca de $30 \%$ dos medicamentos ${ }^{17}$, $70 \%$ das vacinas e $100 \%$ dos soros ofertados pelo SUS ${ }^{18}$. Seus produtos contemplam várias classes terapêuticas, tais quais: antibióticos, ansiolíticos, antiparasitários, antiulcerosos, anticonvulsionantes, anti-helmínticos, antimaláricos e antivirais. Alguns também produzem fitoterápicos e cosméticos ${ }^{4}$. 
Quadro 1. Relação dos LFO por região e tipos de produtos

\begin{tabular}{|c|c|c|c|}
\hline Região & Estado & Instituto & Tipos de produtos \\
\hline \multirow[t]{10}{*}{ Sudeste } & MG & Fundação Ezequiel Dias (Funed) & Medicamentos, soros e vacina \\
\hline & RJ & Instituto Vital Brazil (IVB) & Soros e medicamentos \\
\hline & RJ & $\begin{array}{l}\text { Instituto de Tecnologia em Imunobiológi- } \\
\text { cos (Bio-Manguinhos) }\end{array}$ & $\begin{array}{l}\text { Vacinas bacterianas e virais, kits diagnósti- } \\
\text { cos e medicamentos biofármacos }\end{array}$ \\
\hline & RJ & $\begin{array}{l}\text { Instituto de Tecnologia em Fármacos } \\
\text { (Farmanguinhos) }\end{array}$ & Medicamentos \\
\hline & RJ & $\begin{array}{l}\text { Laboratório Químico Farmacêutico da } \\
\text { Aeronáutica (LAQFA) }\end{array}$ & Medicamentos \\
\hline & RJ & $\begin{array}{l}\text { Laboratório Farmacêutico da Marinha } \\
\text { (LFM) }\end{array}$ & Medicamentos \\
\hline & RJ & $\begin{array}{l}\text { Laboratório Químico Farmacêutico do } \\
\text { Exército (LQFEX) }\end{array}$ & Medicamentos \\
\hline & RJ & Fundação Ataulpho de Paiva (FAP) & Vacina e imunobiológicos \\
\hline & SP & Fundação para o Remédio Popular (Furp) & Medicamentos \\
\hline & SP & Instituto Butantan & Soros e vacinas \\
\hline \multirow[t]{6}{*}{ Nordeste } & BA & $\begin{array}{l}\text { Fundação Baiana de Pesquisa Científica } \\
\text { e Desenvolvimento Tecnológico, Forneci- } \\
\text { mento e Distribuição de Medicamentos } \\
\text { (Bahiafarma) }\end{array}$ & Imunobiológicos e kit diagnóstico \\
\hline & PB & $\begin{array}{l}\text { Laboratório Industrial Farmacêutico do } \\
\text { Estado da Paraíba (Lifesa) }\end{array}$ & Medicamentos \\
\hline & PB & $\begin{array}{l}\text { Instituto de Pesquisa em Fármacos e } \\
\text { Medicamentos (Ipefarm) }\end{array}$ & Medicamentos e insumo farmacêutico \\
\hline & PB & $\begin{array}{l}\text { Laboratório de Avaliação e Desenvol- } \\
\text { vimento de Biomateriais do Nordeste } \\
\text { (Certbio) }\end{array}$ & $\begin{array}{l}\text { Desenvolvimento e avaliação de bioma- } \\
\text { teriais }\end{array}$ \\
\hline & PE & $\begin{array}{l}\text { Laboratório Farmacêutico do Estado de } \\
\text { Pernambuco S.A (Lafepe) }\end{array}$ & Medicamentos \\
\hline & RN & $\begin{array}{l}\text { Núcleo de Pesquisa em alimentos e Medi- } \\
\text { camentos (Nuplam) }\end{array}$ & Medicamentos \\
\hline \multirow[t]{2}{*}{ Sul } & PR & $\begin{array}{l}\text { Centro de Produção e Pesquisa de Imuno- } \\
\text { biológicos (CPPI) }\end{array}$ & Soros, insumos e antígenos \\
\hline & PR & Instituto de Tecnologia do Paraná (Tecpar) & Vacina, insumos e medicamentos \\
\hline \multirow[t]{2}{*}{ Centro-Oeste } & DF & $\begin{array}{l}\text { Empresa Brasileira de Hemoderivados e } \\
\text { Biotecnologia (Hemobrás) }\end{array}$ & Hemoderivados \\
\hline & GO & $\begin{array}{l}\text { Indústria Química do Estado do Goiás } \\
\text { (Iquego) }\end{array}$ & Medicamento e glicosímetro \\
\hline
\end{tabular}

Fonte: elaboração própria, a partir do site da Alfob ${ }^{\mathbf{1 3}}$ e dos portais dos laboratórios.

Entre seus produtos, encontram-se medicamentos e insumos voltados ao tratamento das doenças negligenciadas, que hoje atingem principalmente os países em desenvolvimento. Essas doenças são pouco consideradas para Pesquisa e Desenvolvimento (P\&D) pelas Big Pharmas, visto que são relacionadas com a pobreza e não gerarem retorno financeiro ${ }^{10}$. Neste ponto, destaca-se o papel estratégico desses institutos, que, mesmo de forma tímida, atuam de forma direta ou com parcerias, geralmente com instituições não 
governamentais ou sem fins lucrativos, como a iniciativa de Medicamentos para Doenças Negligenciadas (DNDi - sigla em inglês) na condução de pesquisas para produção de medicamentos e fármacos, novos ou melhorados, para o tratamento dessas enfermidades. Com destaque para a tuberculose, essas doenças são responsáveis por $11,4 \%$ da carga global, entretanto, apenas $1,3 \%$ dos 1.556 novos medicamentos registrados entre 1975 e 2004 foram desenvolvidos especificamente para elas, que, juntas, causam de 500 mil a 1 milhão de óbitos anualmente ${ }^{19}$.

Sob outra vertente, alguns, incluindo Butantan, Bio-Manguinhos e Farmanguinhos, têm atuado no desenvolvimento e/ou produção de produtos de maior complexidade, como os medicamentos biológicos, figurando como instrumentos de desenvolvimento econômico e tecnológico do Ceis. A partir dos incentivos da política industrial, têm contribuído para a redução do preço desses medicamentos e de seu impacto para o orçamento público. Essa nova abordagem já tem trazido resultados positivos para alguns LFO, como aumento do faturamento e maior participação na distribuição de medicamentos especializados e estratégicos ao SUS,20,21.

\section{P\&D E ENSINO}

Os LFO realizam grande parte de suas atividades de $\mathrm{P} \& \mathrm{D}$ de maneira compartilhada, lançando mão de alianças estratégicas com outros laboratórios farmacêuticos e com universidades e centros de pesquisas nacionais e internacionais, disponibilizando suas instalações, equipamentos, materiais e recursos humanos. Esse esforço para inovação pode ser verificado em números. Em pesquisa realizada pela Alfob em 20196, com seus 18 Institutos associados, verificou-se que os LFO investem, em média, $6 \%$ de sua receita em $P \& D$, percentual esse que ainda se mostra tímido quando comparado à média da indústria farmacêutica mundial (aproximadamente $17 \%$ do total de vendas), mas superior à média da indústria farmacêutica brasileira, de 4,8\% ${ }^{22}$. Encontravam-se em andamento: 10 projetos de pesquisa pré-clínica, 3 de pesquisa clínica, e havia 44 pedidos de patentes depositados no Instituto Nacional de Propriedade Intelectual (INPI) ${ }^{6}$.

$\mathrm{Na}$ área de ensino, verificou-se no estudo a atuação de alguns LFO (Funed, Nuplam, Farmanguinhos, Bio-Manguinhos, Butantan) na formação de profissionais, a partir da transferência de conhecimento. Dentre esses, destaca-se Farmanguinhos, que possui uma vice-diretoria de Educação, Pesquisa e Inovação voltada ao preenchimento de lacunas no Sistema Nacional de Inovação em Saúde (SNIS), especialmente no que tange ao desenvolvimento de fármacos e medicamentos, ofertando cursos de atualização, especialização, mestrado e doutorado ${ }^{23}$.

Apesar das iniciativas, por suas origens não estarem associadas à $\mathrm{P} \& \mathrm{D}$, e, sim, à assistência farmacêutica e cobertura das lacunas existentes na produção nacional de vacinas e medicamentos essenciais, verifica-se que os LFO ainda possuem pouca participação no SNIS, comparado a universidades e instituições de pesquisa ${ }^{\mathbf{2 4}}$, sendo necessário que essas instituições avancem em atividades que envolvem inovação, lançando mão, se necessário, de trajetórias incrementais de inovação institucional20.

\section{RECURSOS HUMANOS}

Em relação ao quadro de recursos humanos, com base na comparação entre dois estudos realizados, um em $2004^{24}$ e outro em $2019^{6}$, verifica-se que os LFO têm investido em contratação de pessoal e melhoria na formação dos colaboradores. Nesse período, houve um aumento de $40 \%$ no número de colaboradores, que era de aproximadamente de 5 mil pessoas e passou para 8.352 , no período citado, enquanto o percentual de mestres e doutores passou de $3,6 \%$ para $12,72 \%$, o que mostra um maior foco em ciência. 


\section{INSUMOS FARMACÊUTICOS ATIVOS}

Quanto aos Insumos Farmacêuticos Ativos (IFA), de acordo com uma pesquisa elaborada pela Abifina e Abiquifi, sobre os IFA fabricados no Brasil por seus associados, foi possível verificar que dos 124 IFA identificados, 15 (12\%) estão listados em PDP25. Os números, apesar de pequenos, demonstram a participação dos LFO no esforço do Estado para redução da dependência de insumos e fortalecimento da farmoquímica nacional.

\section{REGULAC̣ÃO DE PREC̣OS}

Destaca-se também o papel desses laboratórios na regulação de preços no mercado nacional. $\mathrm{O}$ superfaturamento de matérias-primas e abusos nos preços de medicamentos têm colocado em evidência o papel regulador do Estado ante o segmento da indústria farmacêutica ${ }^{10}$, e os LFO são instrumentos para essa política. Um exemplo dessa afirmação foi a experiência ocorrida no ano de 2005. Quando o MS anunciou que detinha competência tecnológica para produzir os antirretrovirais Efavirenz, Nelfinavir e Lopinavir, houve uma redução de aproximadamente $50 \%$ nos preços desses medicamentos nos laboratórios privados ${ }^{4}$.

Possuem ainda o intuito de proporcionar economicidade na aquisição de medicamentos e produtos estratégicos pelo SUS, principalmente os de alto custo, comprados anteriormente de empresas privadas, muitas delas estrangeiras, contribuindo para a redução do déficit da balança comercial em saúde, a qual saiu de um patamar de US\$3,0 para US\$ 12 bilhões nos últimos 20 anos, em um período de plena construção do SUS ${ }^{25}$, revelando a forte dependência externa do País em produtos de saúde e a grande vulnerabilidade tecnológica do Ceis.

\section{STATUS E IMPACTO DAS PDP}

As PDP, que são acordos financiados pelo MS para transferência de tecnologia de uma empresa privada a um LFO nacional, contribuíram, em grande parte, para a mudança de perfil dos laboratórios oficiais. Essas parcerias representam, entre outros fatores, o esforço do País para o desenvolvimento da indústria pública de medicamentos e redução da dependência de insumos estrangeiros, visto que o modelo das PDP envolve, além da fabricação do produto por um LFO, a produção do IFA por uma farmoquímica nacional, objetivando a autossuficiência em insumos estratégicos para o Brasil20.

Apesar de todos os desafios políticos e de gestão enfrentados para a conclusão dessas parcerias, verifica-se que, em geral, proporcionaram a modernização ou a criação de plataformas tecnológicas e aprimoramento na capacitação dos profissionais desses institutos, tornando-os aptos a contribuir cada vez mais com a $P \& D$ nacional, alinhada às necessidades do SUS ${ }^{\mathbf{6}, 721}$. Constata-se que favoreceram a incorporação de medicamentos biológicos para alguns LFO, como no caso de Bio-Manguinhos, que, por meio da PDP, deixou de ser um laboratório especializado apenas em vacinas, para a perspectiva de produção de medicamentos biológicos com tecnologias já maduras ${ }^{27}$.

Conforme dados do MS de setembro de 2020 28, existem 91 PDP vigentes. Grande parte dos medicamentos contemplados (42) já possuem registro na Anvisa e já são distribuídos ao SUS (fase III e IV). Abaixo, seguem informações detalhadas sobre as instituições participantes, quantitativos, tipos de produtos, plataforma tecnológica e percentual de PDP por fase (quadro 2) e o total de parcerias, discriminadas por LFO e fase de execução (quadro 3). Verifica-se que os LFO com o maior número de contratos de PDP, Bio-Manguinhos, Farmanguinhos, Butantan e Lafepe, detêm, juntos, $50 \%$ do total, sinalizando que, embora o número de LFO participantes seja grande, a maior parte dos contratos está concentrada em um número reduzido de laboratórios. 
Quadro 2. Retrato das PDP vigentes - set 2020

\begin{tabular}{ll}
\hline Instituições participantes & 18 Instituiç̃es públicas (14 LFO, 2 Universidades, 2 Institutos de Pesquisa) \\
& 32 parceiros privados \\
& 91 vigentes (85 de medicamentos-vacinas-hemoderivados e 6 de produtos para \\
Quande) & 53 medicamentos, 4 vacinas, 6 produtos para saúde e 1 hemoderivado \\
Tipos de Produtos contemplados & $58 \%$ produtos sintéticos 30\% biológicos \\
Plataforma tecnológica & Fase I 9\% / Fase II 45\% \\
Quantitativo por Fase (\%) & Fase III 32\% / Fase IV 14\% \\
\hline
\end{tabular}

Fonte: elaboração própria, com base nas tabelas fornecidas no site do MS. Dados de setembro de $2020^{27}$.

Quadro 3. PDP vigentes por LFO/Fase/Tipo de produto - setembro de 2020

\begin{tabular}{|c|c|c|c|c|}
\hline \multirow[b]{2}{*}{ Total de PDP vigentes/IP } & \multirow[b]{2}{*}{ Instituição Pública (IP) } & \multicolumn{2}{|c|}{ Número de PDP por Fase } & \multirow[b]{2}{*}{ Tipo de produto } \\
\hline & & (I, II, III ou IV) & & \\
\hline \multirow[t]{3}{*}{13} & Farmanguinhos & 4 & ॥ & Sintético \\
\hline & & 6 & III & Sintético \\
\hline & & 3 & IV & Sintético \\
\hline \multirow[t]{5}{*}{13} & Bio-Manguinhos & 1 & । & Biotecnológico \\
\hline & & 6 & $\|$ & \\
\hline & & 4 & III & Biotecnológico \\
\hline & & 1 & & Vacina \\
\hline & & 1 & IV & Biotecnológico \\
\hline \multirow[t]{2}{*}{10} & Butantan & 7 & ॥ & Biotecnológico \\
\hline & & 3 & ॥ा & Vacina \\
\hline \multirow[t]{4}{*}{10} & Lafepe & 1 & I & Sintético \\
\hline & & 4 & $\|$ & Sintético \\
\hline & & 1 & III & Sintético \\
\hline & & 4 & IV & Sintético \\
\hline \multirow[t]{3}{*}{6} & LFM & 2 & I & Sintético \\
\hline & & 3 & III & Sintético \\
\hline & & 1 & IV & Sintético \\
\hline \multirow[t]{2}{*}{6} & IVB & 4 & $\|$ & Sintético \\
\hline & & 2 & IV & Sintético \\
\hline \multirow[t]{2}{*}{6} & Tecpar & 5 & $\|$ & Biotecnológico \\
\hline & & 1 & III & Biotecnológico \\
\hline \multirow[t]{5}{*}{5} & Bahiafarma & 1 & 1 & Produto para saúde \\
\hline & & 1 & & Sintético \\
\hline & & 1 & III & Sintético \\
\hline & & 1 & & Biotecnológico \\
\hline & & 1 & IV & Sintético \\
\hline \multirow[t]{3}{*}{4} & Furp & 2 & ॥ & Sintético \\
\hline & & 1 & III & Sintético \\
\hline & & 1 & ॥ा & Produto para saúde \\
\hline
\end{tabular}


Quadro 3. PDP vigentes por LFO/Fase/Tipo de produto - setembro de 2020

\begin{tabular}{|c|c|c|c|c|}
\hline \multirow[b]{2}{*}{ Total de PDP vigentes/IP } & \multirow[b]{2}{*}{ Instituição Pública (IP) } & \multicolumn{2}{|c|}{ Número de PDP por Fase } & \multirow[b]{2}{*}{ Tipo de produto } \\
\hline & & & & \\
\hline \multirow[t]{2}{*}{4} & Nuplam & 3 & $\|$ & Sintético \\
\hline & & 1 & III & Sintético \\
\hline \multirow[t]{3}{*}{3} & Funed & 1 & $\|$ & Biotecnológico \\
\hline & & 1 & III & Sintético \\
\hline & & 1 & IV & Sintético \\
\hline \multirow[t]{2}{*}{3} & LAQFA & 1 & । & Sintético \\
\hline & & 2 & $\|$ & Sintético \\
\hline \multirow[t]{2}{*}{2} & LQFEX & 1 & । & Sintético \\
\hline & & 1 & III & Sintético \\
\hline 2 & UEPB & 2 & III & Produto para saúde \\
\hline 1 & Hemobrás & 1 & III & Hemoderivado \\
\hline 1 & ICC-Fiocruz/IBMP & 1 & $\|$ & Produto para saúde \\
\hline 1 & Nuplam LAQFA & 1 & $\|$ & Sintético \\
\hline 1 & UFPE & 1 & $\|$ & Produto para saúde \\
\hline 91 & Total & 91 & & \\
\hline
\end{tabular}

Fonte: elaboração própria, baseada nas planilhas fornecidas pela SCTIE/MS no site do MS. Dados de 21/09/202025.

Mesmo diante dos avanços, ameaças vêm sendo enfrentadas para a continuidade das PDP. Como fatores externos aos LFO, podem-se identificar: a falta de estabilidade das políticas de Estado que envolvem os acordos; a instabilidade cambial ocorrida nos últimos anos, que interfere diretamente nos preços negociados; e mais recentemente, em 2019, a suspensão pelo MS de parcerias com $7 \mathrm{LFO}^{29}$, atingindo a produção de 19 medicamentos distribuídos pelo SUS.

\section{FRAGILIDADES E DESAFIOS}

Em que pese o papel estratégico dos LFO para o SUS, apresentam fragilidades, que, muitas vezes, acarretam ociosidade fabril e lentidão de resposta às demandas. Estudos indicam questões como a falta de pessoal qualificado para atividades mais complexas, como para transferência de tecnologia, o que reflete a necessidade de maior intensificação na qualificação de pessoal, voltada às estratégias de inovação. Outros desafios identificados foram rigidez organizacional, planejamento insuficiente, interferência política, entraves relacionados com a burocracia da legislação de compras públicas (Lei n ${ }^{\circ}$ 8.666/93 e Lei $\left.\mathrm{n}^{\mathrm{o}} 10.520 / 02\right)$, limitações de estrutura física, entre outras ${ }^{20,21}$.

Outrossim, dificuldades como corte de investimentos e redução de orçamentos, ocasionadas pelo cenário de congelamento de gastos imposto pela Emenda Constitucional $\mathrm{n}^{0} 95$ e pela atual crise fiscal e financeira do Estado brasileiro, alterações em legislações e acordos, mudanças de pessoal no governo, diminuição de demandas públicas, concorrência desleal com fornecedores estrangeiros e falta de perspectivas de novos concursos públicos ampliam a vulnerabilidade relativa à Covid-19 e ao acesso universal à saúde. Além disso, mudanças trazidas pela 4 a Revolução Tecnológica (big data, mineração de dados, internet das 
coisas etc.), que geram acelerado desenvolvimento tecnológico e científico, trazem desafios adicionais aos institutos, forçando-os a uma revisão de suas competências e de seus tradicionais sistemas de gerenciamentos de dados, a fim de manterem sua competitividade e sustentabilidade $7,19,30,31$.

As vulnerabilidades do Ceis, como exemplo, a dependência externa de insumos e produtos intermediários e as oscilações cambiais, aliadas aos problemas trazidos pela pandemia, como as barreiras protecionistas impostas por países produtores de insumos e medicamentos, como Índia e China; o aumento nos preços de produtos importados usados na produção e pesquisa, devido à elevação da demanda mundial e da desvalorização da moeda nacional; e dificuldades de logística internacional, em função da alta nos preços dos fretes e redução do números de rotas, revelam o difícil cenário atual, que influenciam na capacidade de resposta dos LFO no enfrentamento da pandemia ${ }^{25,31}$.

\section{Avanços na pandemia - ações mais relevantes}

Na pandemia, esforços para o enfrentamento da doença e manutenção das atividades cotidianas vêm sendo realizados pelos LFO. Como exemplos de ações desenvolvidas por alguns deles, verificaram-se em consulta aos seus sítios eletrônicos: produção e distribuição de álcool em gel a profissionais de saúde, aquisição de material de proteção para abastecimento de unidades de saúde, realização de testes diagnósticos, desenvolvimento de máscara resistente ao vírus, ampliação da produção de medicamentos que se encontram em estudo para tratamento da Covid19 e desenvolvimento de soros.

Butantan, Bio-Manguinhos e Farmanguinhos, têm obtido destaque no combate à pandemia, coordenando estudos e ensaios clínicos e realizando parcerias em projetos e pesquisas inovadoras quanto a vacinas e medicamentos.

O Butantan, principal produtor nacional de imunobiológicos e grande fabricante de soros hiperimunes e antígenos vacinais ${ }^{32}$, BioManguinhos, responsável pela autossuficiência em vacinas essenciais do MS e grande produtor de reativos e biofármacos ${ }^{33}$; e Farmanguinhos, maior produtor público de medicamentos antirretrovirais para o $\mathrm{SUS}^{\mathbf{3 4}}$ e com maior número de PDP autorizadas pelo MS ${ }^{21}$, em plena pandemia, intensificaram suas atividades e entregas, realizaram novas alianças internacionais e mantiveram em andamento programas essenciais do MS, como a Vacinação e o Programa DST/Aids, além de cumprirem importantes etapas de suas PDP30,34,35.

\section{BIO-MANGUINHOS E BUTANTAN}

Bio-Manguinhos e Butantan garantiram a produção de vacinas para atendimento do PNI na Campanha de Multivacinação 2020, tendo como destaque a campanha contra o sarampo, cuja vacina tríplice viral (sarampo, caxumba e rubéola) é produzida por Bio-Manguinhos, e a do vírus Influenza (inclui a gripe H1N1), pelo Butantan 32,35.

Em relação à vacina, grandes passos já foram dados em direção ao fortalecimento da ciência e tecnologia nacionais e garantia de autonomia em relação à doença. Dezoito vacinas nacionais estavam em desenvolvimento, em julho de 2021, por universidades e Institutos de Ciência e Tecnologia (ICT), sendo que cinco são de LFO (duas de Bio-Manguinhos e três do Butantan) ${ }^{35}$. No quadro 4, pode-se ver o tipo de vacina e a fase em que se encontram. Caso sejam aprovadas, irão representar um marco em inovação tecnológica para o País, visto que, até hoje, nunca houve uma vacina totalmente nacional, por obstáculos diversos, tais como a insuficiência de políticas para inovação, legislação burocrática, dificuldade em parcerias com a iniciativa privada causada em parte pelo risco do processo e de modo central, pela fragilidade dos produtores nacionais de vacinas em termos de capacitação tecnológica para a inovação, ou seja, capacidade de realizar inovações disruptivas e não apenas incrementais ${ }^{7,37}$. 
Quadro 4. Vacinas nacionais contra Sars-CoV-2 em desenvolvimento - Instituto Farmacêutico Público/Plataforma/Fase de desenvolvimento - dados de julho de 2021

\begin{tabular}{lll}
\hline IFP desenvolvedor & Plataforma tecnológica/Tipo de vacina & Fase de desenvolvimento \\
\hline Bio-Manguinhos & Vacina sintética & Pré-clínica \\
Bio-Manguinhos & Vacina baseada em subunidade proteica & Pré-clínica \\
Butantan/ Dynavax / PATH & Vacina de Vírus inativado & Fase I/II \\
Butantan & Vesículas de membrana externa em plataforma de múlti- & Pré-clínica \\
& plos antígenos & \\
Butantan & Vacina baseada em partículas semelhantes a vírus (VLP) & Pré-clínica \\
\hline
\end{tabular}

Fonte: CGPCLIN/Decit/SCTIE/MS35.

Além do desenvolvimento endógeno de vacinas, outras medidas vêm sendo adotadas por esses LFO para o combate à pandemia e proteção da saúde da população.

O Butantan, ligado ao estado de São Paulo, está desenvolvendo estudos de um soro a partir do plasma de cavalos e de um produto composto por anticorpos monoclonais neutralizantes para seu tratamento ${ }^{38,39}$.

Como ação mais relevante, tem coordenado no Brasil o estudo clínico Proviscov, relacionado com a segurança e a eficácia da vacina produzida pela farmacêutica chinesa Sinovac Biotech. Doze centros de pesquisa foram envolvidos no estudo, abrangendo 9 mil voluntários, todos profissionais de saúde da linha de frente no atendimento a pacientes com a Covid-19. Em setembro de 2020, assinou com a Sinovac um contrato para transferência de tecnologia da vacina contemplando o fornecimento de 46 milhões de doses do imunizante e produção nacional integral, caso os testes do estudo clínico fossem aprovados pela Anvisa. Em janeiro de 2021, o uso emergencial da CoronaVac foi aprovado ${ }^{36,40}$.

Bio-Manguinhos, desde o início da pandemia, tem atuado na contenção e prevenção da doença. Em parceria com o Instituto de Biologia Molecular do Paraná (IBMP) e apoiado pelo Instituto Oswaldo Cruz (IOC/ Fiocruz), o laboratório desenvolveu em tempo recorde o Kit Molecular Sars-CoV-2, que começou a ser distribuído aos Laboratórios Centrais de Saúde Pública (Lacen) e de referência, no início de março, e obteve o registro na Anvisa do Kit TR DPP, resultado de uma parceria com a Chembio (EUA), que, diferentemente, do teste molecular, dispensa estrutura laboratorial e pode ser utilizado em ambulatórios e unidades básicas de atenção primária de saúde ${ }^{41}$.

Outra iniciativa tem sido a avaliação e a discussão de diferentes modelos de parcerias com os principais desenvolvedores mundiais para produção e desenvolvimento clínico em suas instalações ${ }^{42}$. Como resultado, em setembro de 2020, assinou um acordo de encomenda tecnológica com a farmacêutica AstraZeneca, garantindo acesso a 100,4 milhões de doses da vacina ChAdOxl, desenvolvida pela Universidade de Oxford. Assim como o Butantan, o uso emergencial da vacina foi aprovado em janeiro de 2021, e em março, obteve o registro definitivo, tornando-se detentor do primeiro registro de uma vacina Covid-19 produzida no País. Em junho, assinou contrato com a AstraZeneca para a produção $100 \%$ nacionalizada do imunizante, o que será um marco para a produção da vacina no Brasil. O acordo com a AstraZeneca permitirá, além da incorporação tecnológica dessa vacina, o domínio de uma plataforma para desenvolvimento de imunizantes para prevenção de outras enfermidades, como a malária36,42,43.

\section{FARMANGUINHOS}

Quanto aos medicamentos, a estratégia mais rápida que a ciência pode fornecer para ajudar 
no combate à Covid-19 é a análise de fármacos já aprovados para outros usos ${ }^{44}$.

Farmanguinhos tem apoiado as pesquisas desenvolvidas para o tratamento da Covid-19 com fornecimento de medicamentos e placebos. Dois de seus medicamentos tiveram sua eficácia testada. Para estudos do antimalárico cloroquina, colaborou com o ensaio clínico Solidarity, da Organização Mundial da Saúde (OMS), coordenado pela Fiocruz com a participação de Bio-Manguinhos; e com o estudo CloroCOVID-19, desenvolvido pela Fundação de Medicina Tropical Dr. Heitor Vieira Dourado, em Manaus (AM) ${ }^{45}$. Quanto ao antirretroviral Atazanavir, tem contribuído com o estudo coordenado pelo Centro de Desenvolvimento Tecnológico em Saúde (CDTS), com envolvimento de cientistas da Fiocruz, Instituto D'Or de Pesquisa e Ensino e da Universidade Iguaçu ${ }^{46}$ e com a intitulada Coalizão Covid-19 Brasil IX, em parceria com a farmacêutica Blanver e o Hospital do Coração (HCor). Cabe destacar que nenhum desses medicamentos se mostrou promissor até o momento. No entanto, o LFO tem estudado a viabilidade de pesquisa e desenvolvimento de novos fármacos e buscado parcerias para outros produtos ${ }^{47}$.

\section{Conclusões}

Os LFO, tanto em iniciativas já estabelecidas como em ações prospectivas, têm explicitado suas competências para o desenvolvimento econômico e tecnológico do Ceis, as quais têm se manifestado no enfrentamento da pandemia. Suas atividades demonstram o esforço para integração das políticas públicas de saúde e industriais, tanto no âmbito federal como no estadual. Com a mudança de perfil, promovida pela necessidade de competitividade e fortalecimento da capacidade científica e produtiva, muitos migraram de um modelo puramente fabril para um mais estratégico, desempenhando cada vez mais o papel de ICT.
Por suas atividades e estratégias durante a pandemia, identificadas no estudo, analisando-se de uma perspectiva mais ampla, foi possível perceber o grande papel que esses institutos desempenham para manutenção dos diversos programas do MS e redução das vulnerabilidades do Ceis. Nesse cenário, reitera-se a importância da ampliação do papel do Estado para manutenção e sustentação de um ambiente que favoreça o sistema de inovação em saúde, essencial para o desenvolvimento econômico do País e sustentabilidade de sua indústria farmacêutica.

Em um cenário de transformação em curso, ocasionado pela $4^{\mathrm{a}}$ Revolução Tecnológica, impõe-se a esses institutos uma agenda de mudança. Para alcance de sustentabilidade e desenvolvimento em longo prazo, devem investir continuamente na cooperação com instituições públicas e privadas, na qualificação de seus profissionais e na incorporação de novos modelos de gestão e tecnologias. $\mathrm{O}$ compromisso com a produção de medicamentos e produtos de saúde essenciais para o SUS, envolvendo desde os mais básicos até os de maior complexidade e valor agregado, assim como o incremento de atividades associadas à inovação, são medidas fundamentais para fortalecimento do braço estratégico público do Ceis e maior suporte ao SUS, diante de seu enorme desafio de garantia de acesso universal à saúde.

\section{Colaboradores}

Fernandes DRA (0000-0002-0969-2707)* contribuiu para a concepção, planejamento, coleta de dados, interpretação dos resultados e redação do trabalho. Gadelha CAG (00000002-9148-8819)* contribuiu para a supervisão do estudo, análise, interpretação dos resultados e revisão crítica do manuscrito. Maldonado JMSV (0000-0002-0815-1765)* contribuiu para a análise, interpretação dos resultados e revisão crítica do manuscrito. 


\section{Referências}

1. Oliveira MA, Bermudez JAZ, Osório-de-Castro CGS. Assistência farmacêutica e acesso a medicamentos. Rio de Janeiro: Editora Fiocruz; 2007. 112 p.

2. Gadelha CAG, Costa LS, Maldonado J. O complexo econômico-industrial da saúde e a dimensão social e econômica do desenvolvimento. Rev. Saúde Púb. 2012 [acesso em 2020 dez 20]; 46(supl1):2128. Disponível em: https://doi.org/10.1590/S003489102012005000065 .

3. Giovanella L, Mendoza-Ruiz A, Pilar ACA, et al. Sistema universal de saúde e cobertura universal: desvendando pressupostos e estratégias. Ciênc. Saúde Colet. 2018 [acesso em $2020 \mathrm{dez}$ 20]; 23(6):17631776. Disponível em: https://doi.org/10.1590/141381232018236.05562018 .

4. Magalhães JL, Antunes MAS, Andrade NB. Laboratórios farmacêuticos oficiais e sua relevância para saúde pública do Brasil. RECIIS, Rev. Eletrônica Comum. 2011 [acesso em $2020 \mathrm{dez}$ 20]; 5(1):85-99. Disponível em: https://doi.org/10.3395/reciis.v5i1.512.

5. Bermudez JAZ, Esher A, Osorio-de-Castro CGS, et al. Assistência Farmacêutica nos 30 anos do SUS na perspectiva da integralidade. Ciênc. Saúde Colet. 2018 [acesso em $2020 \mathrm{dez} 20$ ]; 23(6):1937-1949. Disponível em: https://dx.doi.org/10.1590/141381232018236.09022018 .

6. Associação dos Laboratórios Farmacêuticos Oficiais do Brasil. Conselho Federal de Farmácia. Laboratórios farmacêuticos oficiais do Brasil. Brasília, DF: Alfob/CFF; 2019 .

7. Almeida ÁCS. Análise dos efeitos das parcerias para o desenvolvimento produtivo nos laboratórios farmacêuticos oficiais. [tese]. Rio de Janeiro: Universidade Federal do Rio de Janeiro; 2018. 318 p.

8. Fundação Oswaldo Cruz, Instituto de Tecnologia em Fármacos Farmanguinhos. História 1976 a 1989. [acesso em 2020 dez 20]. Disponível em: https://www.far. fiocruz.br/instituto/historia/1976-a-1990/.
9. Fundação Oswaldo Cruz, Instituto de Tecnologia em Imunobiológicos. História. Rio de Janeiro: Fiocruz; 2019. [acesso em 2020 dez 20]. Disponível em: https://www.bio.fiocruz.br/index.php/br/home/historia.

10. Oliveira EA, Labra ME, Bermudez J. A produção pública de medicamentos no Brasil: uma visão geral. Cad. Saúde Pública. 2006; 22(11):2379-2389.

11. Fundação Oswaldo Cruz, Instituto de Tecnologia em Fármacos Farmanguinhos. Relatório de atividades 2009-2013. Rio de Janeiro: Fiocruz; 2014.

12. Chaves GC, Oliveira MA. Estratégias governamentais para redução de preços de medicamentos em situação de monopólio: produção local e enfrentamento da barreira patentária. In: Hasenclever L, Paranhos J, Chaves G, et al. Vulnerabilidades do complexo industrial da saúde: reflexos das políticas industrial e tecnológica na produção local e assistência farmacêutica. Rio de Janeiro: E-papers; 2018. p. 181-211.

13. Agência Nacional de Vigilância Sanitária. Consultas. 2021. [acesso em 2021 maio 16]. Disponível em: https://consultas.anvisa.gov.br.

14. Ferreira Neto PTP, Cunha CR. Produção pública de medicamentos no Brasil no contexto da pandemia da COVID-19. Rev. Gest. Saúde (Brasília). 2020 [acesso em 2020 dez 20]; 11(3):296-309. Disponível em: https://periodicos.unb.br/index.php/rgs/article/view/32291.

15. Brasil. Ministério da Saúde, Secretaria de Ciência, Tecnologia, Inovação e Insumos Estratégicos em Saúde. Relação Nacional de Medicamentos Essenciais: Rename 2020. Brasília, DF: Ministério da Saúde; 2020. 217 p. [acesso em 2020 dez 2]. Disponível em: https://bvsms.saude.gov.br/bvs/publicacoes/relacao_medicamentos_rename_2020.pdf.

16. Vieira FS, Santos MAB. Texto para discussão. O setor farmacêutico no Brasil sob as lentes da conta-satélite de saúde. Brasília, DF: Rio de Janeiro: Ipea; 2020. 
17. Brasil. Ministério da Saúde. Laboratórios Oficiais. 2018. [acesso em 2020 set 2]. Disponível em: https:// www.saude.gov.br/assistencia-farmaceutica/laboratorios-oficiais.

18. Associação dos Laboratórios Farmacêuticos Oficiais do Brasil. Laboratórios associados. 2020. [acesso em 2020 set 2]. Disponível em: http://alfob.org.br/laboratorios-associados/.

19. Mendonça J. Importância de laboratórios oficiais para a sustentabilidade de políticas públicas e o desenvolvimento tecnológico no país. Rev. Facto Abifina. $2018 ; 12(57): 28-31$.

20. Rezende KS. As parcerias para o desenvolvimento produtivo e estímulo à inovação em instituições farmacêuticas públicas e privadas. [dissertação]. Rio de Janeiro: Escola Nacional de Saúde Pública Sergio Arouca, Fundação Oswaldo Cruz; 2013. [acesso em 2017 out 10]. Disponível em: http://bases.bireme. br/cgi-bin/wxislind.exe/iah/online/?IsisScript=iah/ iah.xis\&src $=$ google \&base $=$ LILACS\&lang $=p \& n e x t A c$ tion=lnk\&exprSearch=688814\&indexSearch=ID .

21. Fernandes DRA. Fatores críticos de sucesso em Parceria para o Desenvolvimento Produtivo PDP Estudo de caso em um laboratório farmacêutico público (Farmanguinhos). [dissertação]. Rio de Janeiro: Escola Nacional de Saúde Pública Sergio Arouca, Fundação Oswaldo Cruz; 2019. [acesso em 2020 nov 10]. Disponível em: https://www.arca.fiocruz.br/handle/ icict/34235.

22. Akkari ACS, Munhoz IP, Tomioka J, et al. Inovação tecnológica na indústria farmacêutica: diferenças entre a Europa, os EUA e os países farmaemergentes. Gest. Prod. 2016 [acesso em 2020 set 10]; 23(2):365380. Disponível em: https://doi.org/10.1590/0104$-530 \times 2150-15$.

23. Fundação Oswaldo Cruz. Educação Farmanguinhos. Guia para Estudantes. Rio de Janeiro: Fiocruz; 2019. [acesso em 2021 set 10]. Disponível em: https://www. far.fiocruz.br/wp-content/uploads/2019/05/guia-do-estudante.pdf.
24. Bastos VD. Laboratórios farmacêuticos oficiais e doenças negligenciadas: perspectivas de política pública. Revista do BNDES. 2006; 13(25):[269]-298.

25. Associação Brasileira das Indústrias de Química Fina, Biotecnologia e suas Especialidades. A Hora da virada para a indústria farmoquímica. Rev. Facto Abifina. 2020 [acesso em 2020 nov 15]; 14(63):22-25. Disponível em: http://www.abifina.org.br/revista_facto_ home.php.

26. Gadelha CAG, Temporão JG. Desenvolvimento, Inovação e Saúde: a perspectiva teórica e política do Complexo Econômico-Industrial da Saúde. Ciênc. Saúde Colet. 2018 [acesso em 2020 nov 15]; 23(6):18911902. Disponível em: https://doi.org/10.1590/141381232018236.06482018

27. Costa NR, Lago RF, Souza ACA, et al. Complexo Econômico-Industrial da Saúde e a produção local de medicamentos: estudo de caso sobre sustentabilidade organizacional. Saúde debate. 2019; 43(esp7):8-21.

28. Brasil. Ministério da Saúde. Parcerias para o Desenvolvimento Produtivo (PDP). Brasília, DF: Ministério da Saúde; 2020. [acesso em 2020 set 23]. Disponível em: https://saude.gov.br/saude-de-a-z/parcerias-para-o-desenvolvimento-produtivo-pdp.

29. Matos A. Fiocruz esclarece sobre contratos de PDP. Rio de Janeiro: Fundação Oswaldo Cruz, Instituto de Tecnologia em Fármacos Farmanguinhos; 2019. [acesso em 2020 dez 10]. Disponível em: https://www. far.fiocruz.br/2019/07/fiocruz-esclarece-sobre-contratos-de-parcerias-de-desenvolvimento-produtivo$-\mathrm{pdp} /$.

30. Fundação Oswaldo Cruz. Relatório de atividades 2020. Rio de Janeiro: Fiocruz; 2020.

31. Fernandes DRA, Gadelha CAG, Maldonado JMSV. Vulnerabilidades das indústrias nacionais de medicamentos e produtos biotecnológicos no contexto da pandemia de COVID-19. Cad. Saúde Pública. 2021; 37(4):e00254720.

32. Instituto Butantan. O Instituto. São Paulo. [acesso 
em 2020 nov 20]. Disponível em: https://butantan. gov.br/institucional/o-instituto.

33. Fundação Oswaldo Cruz, Instituto de Tecnologia em Imunobiológicos. Quem somos. Rio de Janeiro: Fiocruz, Bio-Manguinhos; 2020. [acesso em 2020 nov 20]. Disponível em: https://www.bio.fiocruz. br/index.php/br/home/quem-somos\#: :text=O\%20 Complexo\%20 Tecnol\%C $3 \%$ B 3 gico\% 20 de\%20Vacinas,Minist\%C3\%A 9rio\%20da\%20 Sa\%C3\%BAde\%20(MS).

34. Oliveira V. Farmanguinhos obtém registro de mais um antirretroviral. Rio de Janeiro: Fiocruz, Farmanguinhos; 2020. [acesso em 2020 nov 20]. Disponível em: https://www.far.fiocruz.br/2020/10/farmanguinhos-obtem-registro-de-mais-um-antirretroviral/.

35. Schueler P. Bio-Manguinhos fornece vacinas para Campanha de Multivacinação. Bio-Manguinhos. Rio de Janeiro: Bio-Manguinhos, Fiocruz; 2020. [acesso em 2020 out 19]. Disponível em: https://www.bio. fiocruz.br/index.php/br/noticias/2016-bio-manguinhos-fornece-vacinas-para-campanha-de-multivacinacao.

36. Brasil. Ministério da Saúde, Secretaria de Ciência, Tecnologia, Inovação e Insumos Estratégicos em Saúde. Relatório Técnico - Monitoramento de vacinas em desenvolvimento contra SARS-CoV-2. Brasília, DF: Ministério da Saúde; 2020.

37. Bohem C. Butantan desenvolve a primeira vacina nacional contra covid-19. Agência Brasil. 2021 mar 16. [acesso em 2021 ago 20]. Disponível em: https:// agenciabrasil.ebc.com.br/saude/noticia/2021-03/butantan-desenvolve-primeira-vacina-nacional-contra-covid-19.

38. Instituto Butantan. Soro contra a Covid-19 produzido pelo Butantan está pronto para ser testado em humanos. São Paulo. 2021. [acesso em 2021 mar 6]. Disponível em: https://butantan.gov.br/noticias/soro-contra-a-covid-19-produzido-pelo-butantan-esta-pronto-para-ser-testado-em-humanos.
39. Instituto Butantan. Fapesp: Butantan vai desenvolver, em laboratório, anticorpos para tratamento. São Paulo: Agência Fapesp; 2020. [acesso em 2020 set 30]. Disponível em: http://www.butantan.gov.br/noticias/fapesp-butantan-vai-desenvolver-em-laboratorio-anticorpos-para-tratamento-da-covid-19.

40. Instituto Butantan. Governo de SP assina contrato com Sinovac para o fornecimento de 46 milhões de doses da Coronavac. São Paulo. 2020. [acesso em 2020 out 3]. Disponível em: https://butantan.gov.br/ noticias/governo-de-sp-assina-contrato-com-sinovac-para-o-fornecimento-de-46-milhoes-de-doses-da-coronavac.

41. Instituto de Biologia Molecular do Paraná. Pesquisadores desenvolvem Kit diagnóstico para o novo coronavírus. Paraná: IBMP; 2020. [acesso em 2020 ago 2]. Disponível em: http://www.ibmp.org.br/pt-br/pesquisadores-do-ibmp-e-da-fiocruz-desenvolvem-kit-diagnostico-para-o-novo-coronavirus/.

42. Fundação Oswaldo Cruz. Fiocruz assina contrato de 100 milhões de doses da vacina. Rio de Janeiro: Fiocruz; 2020. [acesso em 30 set 2020]. Disponível em: https://portal.fiocruz.br/noticia/fiocruz-assina-contrato-de-100-milhoes-de-doses-da-vacina.

43. Fundação Oswaldo Cruz. Fiocruz recebe primeiro registro da Anvisa para vacina Covid-19 produzida no Brasil. Rio de Janeiro: Fiocruz; 2021. [acesso em 2021 jul 10]. Disponível em: https://portal.fiocruz.br/ noticia/fiocruz-recebe-primeiro-registro-da-anvisa-para-vacina-covid-19-produzida-no-brasil.

44. Fundação Oswaldo Cruz. Fiocruz investiga ação de antirretrovirais contra Covid-19. Rio de Janeiro: Fiocruz; 2020. [acesso em 2020 maio 8]. Disponível em: https://portal.fiocruz.br/noticia/fiocruz-investiga-acao-de-antirretrovirais-contra-covid-19.

45. Brasil. Ministério da Saúde. Ministério da Saúde acompanha 9 estudos para obter novos tratamentos contra coronavírus. Brasília, DF: Agência Saúde; 2020. [acesso em 2020 maio 7]. Disponível em: https:// www.saude.gov.br/noticias/agencia-saude/46669- 
-ministerio-da-saude-acompanha-9-estudos-para-obter-novos-tratamentos-contra-coronavirus.

46. Assessoria de Comunicação. Farmanguinhos participa de estudos clínicos da Cloroquina. Rio de Janeiro: Fiocruz, Farmanguinhos; 2020. [acesso em 2020 maio 7]. Disponível em: https://www.far.fiocruz.br/2020/04/cloroquina/.

47. Sandes T. Quatro projetos de Farmanguinhos são aprovados para o Inova Fiocruz Covid-19. Rio de Ja- neiro: Fiocruz, Farmanguinhos; 2020. [acesso em 2021 ago 2]. Disponível em: https://www.far.fiocruz. br/2020/07/quatro-projetos-de-farmanguinhos-sao-aprovados-para-o-inova-fiocruz-covid-19/.

Recebido em 10/06/2021

Aprovado em 12/10/2021

Conflito de interesses: inexistente

Suporte financeiro: Projeto 'Desafios para o Sistema Único de

Saúde no contexto nacional e global de transformações sociais,

econômicas e tecnológicas - CEIS 4.0' 